\title{
Drug Sensitivity and Molecular Diversity of $M$. tuberculosis in Cameroon: A Meta-analysis
}

Titanji VPK ${ }^{1 *}$ and Assam JPA ${ }^{1,2}$

${ }^{1}$ Biotechnology Unit, Faculty of Science, University of Buea, Buea, Cameroon

${ }^{2}$ Laboratory for Tuberculosis Research, CANTAM TB project Biotechnology center of Nkolbisson, Faculty of Science, University of Douala, Douala, Cameroon

\begin{abstract}
Tuberculosis (TB) represents one of the most challenging threats to global human health. $M$. tuberculosis causes about 8.8 million new cases of active tuberculosis and 1.1 million deaths annually. According to Cameroon's National TB Control Program (NTBCP), tuberculosis still remains a major public health problem: an important cause of mortality and morbidity, with a negative impact on the socio-economic condition of the population. This paper is a meta-analysis review of the molecular diversity and drug sensitivity trends to first line TB drugs and the threat of multi-drug resistant (MDR) strains to the TB control programs in Cameroon. A very large selection of papers from the last 20 years was done through Google, Google scholar, Pub Med using as keys words 'Mycobacterium tuberculosis, tuberculosis, resistance to first and second line drugs, molecular typing, Cameroon'. The paper to be selected for analysis must have dealt with at least one of the key words and must have been carried out in at least one of the regions of Cameroon. The selected paper was red and the required information for the review was then extracted. Investigations of genetic polymorphism of $M$. tuberculosis complex strains from humans in Cameroon has shown M. tuberculosis sensu stricto to be the predominant agent of TB cases, with the preponderance of the LAM10 family genotype and large shift of $M$. africanum. The resistance to all first line anti-TB drugs has declined significantly, however, the general rates of anti-TB drug resistance remain high in some regions, underscoring the need for greater enforcement of control strategies in the country.
\end{abstract}

Keywords: Mycobacterium tuberculosis; Tuberculosis; Resistance; Molecular typing; Cameroon

\section{Introduction}

Tuberculosis (TB) represents one of the most challenging threats to global human health. M. tuberculosis causes about 9 million new cases of active tuberculosis and 1.5 million deaths annually [1]. Moreover, it is estimated that over one-third of the world's population has latent tuberculosis infection, which represents a huge reservoir for the disease. Exponential development of travel extends this threat worldwide [1]. Ninety-five percent of cases occur in developing countries, where the lack of proper health care systems leads to incomplete case detection and treatment. The high prevalence of Human Immune Virus (HIV), which weakens the immune system, favors the spread of latent TB infection. The emergence and spread of resistance to first line TB drugs has rendered the control of the disease more difficult in sub-Saharan Africa [1].

Cameroon, a country with 20 million inhabitants has been classified as a moderate tuberculosis burden country according to the World Health Organization (WHO) [1]. According to Cameroon's National TB Control Program (NTBCP), tuberculosis still remains a major public health problem: an important cause of mortality and morbidity, with a negative impact on the socio-economic condition of the population. According to the National TB Control Program 26110 tuberculosis cases were recorded, representing an increase rate of $3 \%$ of total cases in 2013 against 2012 that registered 25360 cases despite the implementation of the directly observed treatment short course strategy (DOTs) [2]. This paper is a meta-analysis review of the molecular diversity and drug sensitivity trends to first line TB drugs and the threat of multi-drug resistant (MDR ) strains to the TB control programs in Cameroon.

\section{Methodology}

A very large selection of papers from the last 20 years was done through Google, Google scholar, Pub Med using as keys words 'Mycobacterium tuberculosis, tuberculosis, resistance to first and second line drugs, molecular typing, Cameroon'. The paper to be selected for analysis must have dealt with at least one of the key words and must have been carried out in at least one of the regions of Cameroon. The selected paper was red and the required information for the review was then extracted.

\section{Mycobacterium Species Infecting Humans in Cameroon}

Tuberculosis is an infectious and contagious disease caused by Mycobacterium (M.) tuberculosis Complex(Mycobacterium tuberculosis, Mycobacterium africanum, Mycobacterium bovis, Mycobacterium microti and Mycobacterium canetti). Many studies have been conducted in Cameroon to identify the species of the Mycobacterium tuberculosis complex (MTBC) responsible for pulmonary tuberculosis. In the 1970 s, one study performed 30 years ago reported that $56 \%$ of cases of TB were due to Mycobacterium africanum (M. africanum) strains in Cameroon and $44 \%$ of cases were due to others species (M. tuberculosis and M. bovis) [3]. A subsequent study conducted by Niobe-Eyangoh et al. in 2003 [4] using the similar methods used described by Huet et al. [3], analyzed the biodiversity of $M$. tuberculosis complex isolates collected from the population of the West Region, showed that only

*Corresponding author: Titanji VPK, Biotechnology Unit, Faculty of Science, University of Buea, P.O. Box 63, Buea, Cameroon, Tel: (237) 998674 54; E-mail: vpk.titanji@yahoo.com

Received January 08, 2016; Accepted April 25, 2016; Published May 03, 2016

Citation: Titanji VPK, Assam JPA (2016) Drug Sensitivity and Molecular Diversity of $M$. tuberculosis in Cameroon: A Meta-analysis. J Vasc Med Surg 4: 266 doi:10.4172/2329-6925.1000266

Copyright: @ 2016 Titanji VPK, et al. This is an open-access article distributed under the terms of the Creative Commons Attribution License, which permits unrestricted use, distribution, and reproduction in any medium, provided the original author and source are credited. 
$9 \%$ of strains were M. africanum. Despite the use of more sensitive and specific genotyping methods only $9 \%$ of the isolates were classified as M. africanum. This suggested that the decreasing trend observed could not be attributed to identification bias but was in fact due to a genuine regression of $M$. africanum as the etiologic agent of TB in Cameroon in the last 3 decades (from 56 to 9\%). Also from that study they found that, despite the high prevalence of bovine TB in Cameroon [5], pulmonary TB caused by $M$. bovis in humans remains rare, thus only 41 (9\%) of the 455 M. tuberculosis complex strains were M. africanum, while 413 were $M$. tuberculosis, and 1 was $M$. bovis [4]. A more recent study done in Centre and West regions of Cameroon by AssamAssam et al. showed by Molecular analysis that all TB cases reported in this study were caused by infections with $M$. tuberculosis $(98.8 \%$; 167/169) while Mycobacterium africanum had dwindled to $(1.2 \%$; $2 / 169$ ) respectively and no $M$. bovis was detected [6]. This latter study also indicated a shift in type of infectious agent $M$. africanum versus M. tuberculosis [6]. A study conducted by Kamgue et al. in the Centre region of Cameroon showed that on the 298 isolates analyzed, 291 (97.65\%) were classified as Mycobacterium tuberculosis and 6 (2.03\%) as Mycobacterium africanum species. The remaining one isolate was identified as Mycobacterium bovis [7]. This latest also confirmed the regression of M. africanum as the etiologic agent of TB in Cameroon and demonstrates that pulmonary TB caused by M. bovis in humans remains rare. The low rate of human pulmonary TB caused by $M$. bovis in these studies could be explained by different factors: (i) a high number of the M. bovis infections are responsible for extra-pulmonary $\mathrm{TB}$ cases and in these studies the TB cases were essentially pulmonary cases or (ii) pulmonary TB due to M. bovis may be more frequent in rural areas [7]. However, the majority of the patients in these studies were from urban areas like Yaounde, Mbalmayo and Bafoussam. Thus it is evident that, M. tuberculosis, M. africanum and M. bovis remain the MTBC species causing pulmonary TB in Cameroon with a large predominance of $M$. tuberculosis, a large regression of $M$. africanum and a rarity of $M$. bovis.

Bovine tuberculosis (bTB) is an important neglected zoonosis in Cameroon, where many communities depend on their livestock for livelihood and the incidence of human TB and TB-HIV/AIDS coinfection are high and increasing annually [8]. It is mainly caused by Mycobacterium bovis which can also cause disease in human and in a variety of domestic and wild animals [8]. A study done by Ndukum et al. in littoral and West region of Cameroon showed that TB lesions are common pathologies identified in slaughtered cattle and rates ranging from $0.18 \%$ to $4.25 \%$ have been recorded $[9,10]$. Another study done by Ndukum et al. in the Adamaoua region and North West region of Cameroon to estimate the prevalence of bovine TB in cattle in the highlands of Cameroon. showed that bovine TB is prevalent in live cattle and meat production abattoirs in Cameroon. Furthermore, bovine TB was found to be widely distributed in live cattle (4.67-5.44\%) and was higher in the Western highlands than Adamawa plateau [11]. A survey by Koro Koro et al. in Littoral and Centre regions showed a prevalence of $0.81 \%$ and $1.3 \%$ of bovine tuberculosis in Yaoundé and Douala abattoirs respectively. This result show that bovine tuberculosis can be still prevalent at low levels in cattle destined for human consumption in Cameroon and highlighted the importance of rigorous veterinary inspection of animals destined for human consumption. [12].

\section{Evolution of TB Cases in Cameroon}

In Cameroon, a country with 20 million inhabitants, the incidence rate of TB is estimated to have increased from 77 cases per 100,000 inhabitants in 2000 to 91 cases per 100,000 inhabitants in 2004 [13]. Figure 1 shows an extended bell shaped prevalence graph with TB increasing over the decade from 1990 to 2000 levelling between 2002 and 2004 and tending to drop thereafter. This trend reversal can be explained at least in part by the institution of the NTBCP in 2004. We note an increase until the year 2006 with a trend to stabilization since than: The number of sm+ pulmonary TB cases notified represents about $95 \%$ of the estimated cases [14]. On the contrary, there is a detection gap of more than $50 \%$ with regard to sm- and extra-pulmonary TB cases according to WHO estimations [14]. In 2013 26,110 new sputum smear-positive cases were reported, even if it has stabilize since 2008 we can notice that the number of cases is still high (Figure 1).

A better view of this number of cases per regions as shown by Table 1 based on the according to the National Control TB Program (NCTP) report of 2013. As we can notice, this results obtained per region is very high and has increase the number of Center for Diagnosis and Treatment (CDT). Actually the NCTP has 216 CDT distributed in the 10 regions of the country (Table 1 ).

\section{Molecular Typing of Tb Isolates in Cameroon}

In recent years, molecular typing methods have become useful tools in epidemiological studies for the control of TB, and have revealed insights into the population structure of clinical isolates in different geographical locations. Two of the most useful typing methods are spoligotyping [15] based on polymerase chain reaction (PCR) amplification of a highly polymorphic direct repeat locus in the $M$. tuberculosis genome, and mycobacterial interspersed repetitiveunit-variable-number tandem-repeat (MIRU-VNTR) [16], which uses length polymorphisms of mini-satellite-like loci in the genome. Spoligotyping targeting the direct repeat locus is a rapid, simple, and cost-effective system that allows the simultaneous detection and differentiation of M. tuberculosis complex (MTBC) strains and provides genotypic information [15]. It is a good indicator of strain identity

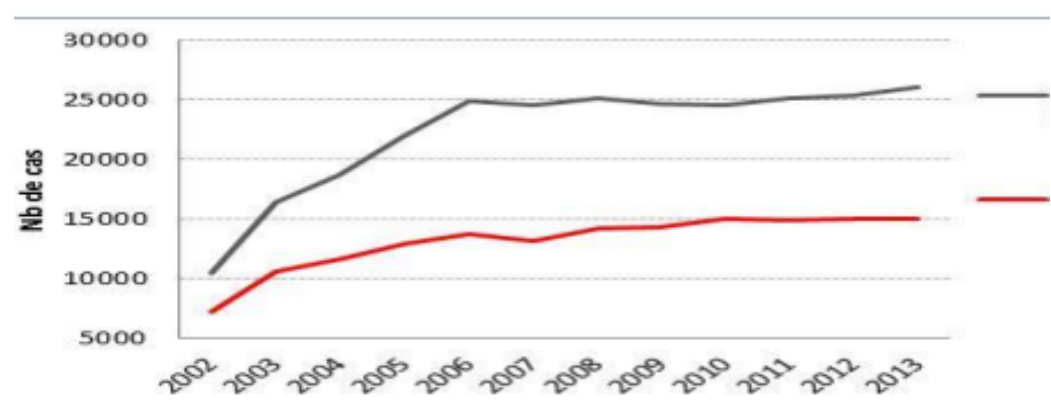

All TB cases

New TB cases

Figure 1: Prevalence of Tuberculosis (rate for 100,000 inhabitants) [14]. 


\begin{tabular}{|c|c|c|}
\hline $\begin{array}{c}\text { Regions of } \\
\text { Cameroon }\end{array}$ & $\begin{array}{c}\text { Number of TB cases, all forms } \\
\text { detected }\end{array}$ & $\begin{array}{c}\text { Rate for } \mathbf{1 0 0} \mathbf{0 0 0} \\
\text { inhabitants }\end{array}$ \\
\hline Adamaoua & 1526 & 138 \\
\hline Centre & 7565 & 283 \\
\hline East & 1793 & 214 \\
\hline Extreme North & 5393 & 90 \\
\hline Littoral & 6013 & 331 \\
\hline North & 2078 & 91 \\
\hline North West & 1701 & 89 \\
\hline West & 1293 & 69 \\
\hline South & 892 & 122 \\
\hline South West & 1856 & 127 \\
\hline Total & 26110 & 125 \\
\hline &
\end{tabular}

Table 1: Statistics of Tuberculosis cases in 2013.

and provides information about epidemiologically important clones [17]. Another advantage of spoligotyping is its ability to measure the overall diversity of $M$. tuberculosis complex strain patterns, including differences between regions and populations and the prevalence of endemic strains $[18,19]$. MIRU-VNTR typing is technically flexible, as sizing can be done using capillary [20,21] or gel electrophoresis [22] or non-denaturing high-performance liquid chromatography [23]. It is one of the most promising PCR-based methods for detecting the number of tandem repeats at a given genetic locus. Supply et al. [24] defined a set of 15 MIRU-VNTR loci for molecular epidemiological investigations and a set of 24 MIRU-VNTR loci for phylogenetic analysis of $M$. tuberculosis strains worldwide. In support of this, another study concluded that this "real-time" MIRUVNTR genotyping approach was highly applicable for population based studies [25]. This view was reinforced by a study conducted in the Brussels region, where the authors concluded that a standardized MIRU-VNTR genotyping method could be a new reference for epidemiological and phylogenetic screening of $M$. tuberculosis strains because it is useful to confirm spoligotyping clusters or to discriminate among the isolates that they contain [26].

Ealry work on the molecular typing of TB isolates in Cameroon was done by Sara Eyangoh et al. from 455 M. tuberculosis complex strains collected in West region. Spoligotyping of the 413 M. tuberculosis isolates revealed 72 distinct spoligotypes. Thirty-five unique spoligotype patterns were seen, and the remaining 375 isolates grouped into 37 clusters. Most of the clusters contained only two strains (15 clusters). One cluster included 140 isolates. Overall, the clustering rate was much higher among $M$. tuberculosis strains (91\%) than among $M$. africanum strains (66\%). These results reflect the presence of a predominant genetic group in Cameroon, including $31 \%$ of the $M$. tuberculosis complex strains, and show transmission of an endemic strain. The other strains were genetically heterogeneous, showing high local diversity. The main M. tuberculosis cluster (140 isolates) corresponded to spoligotype 61 . The second-largest group corresponded to the ubiquitous Haarlem family (spoligotypes 50 and 47 ), which represents $11 \%$ of all $M$. tuberculosis complex strains. The African-type M. tuberculosis (spoligotype 52), as defined by Kremer et al. [17], represented $5 \%$ of all M. tuberculosis complex strains. Finally, $4.5 \%$ of the $M$. tuberculosis strains were the ubiquitous spoligotype 53. None of the strains had the spoligotype of the worldwide expanded epidemic Beijing family. The most prevalent spoligotype was 61 , which was shown by isolates from 140 patients. This spoligotype represents $34 \%$ of all $M$. tuberculosis isolates. The specific characteristic of spoligotype 61 is the lack of spacers 23,24, and 25 in the DR region [4]. Cameroon family M. tuberculosis strains (i) are part of the major genetic group 2 and lack the TbD1 region like other families of epidemic strains, (ii) lack spacers 23, 24, and 25 in their direct repeat (DR) region, (iii) have an identical number of repeats in 8 of 12 variable-number tandem repeats of mycobacterial interspersed repetitive unit (MIRU-VNTR) loci, (iv) have similar IS6110-restriction fragment length polymorphism (RFLP) multiband patterns (10 to 15 copies) with seven common IS6110 bands, (v) do not have an IS6110 element in their DR locus, and (vi) have four IS6110 elements in open reading frames (adenylate cyclase, phospholipase $\mathrm{C}, \mathrm{moe} Y$, and ATP binding genes) [27]. Their findings confirm the recent expansion of this family in Cameroon and indicate that the interpretation of molecular typing results has to be adapted to the characteristics of the strain population within each setting.

Subsequent work on molecular typing of TB isolates collected from Yaoundé was done by Penlap et al. in 2010 has suggested a preponderance of LAM10_CAM using spoligotyping method and also 8 new spoligotypes were discovered. There results present also a predominance resistant phenotypes among the LAM10_CAM family [28].

In 2013 a work done in the savannah mosaic zone (West and North West regions) and the tropical rainforest zone (Central region) by Assam-Assam et al. showed that most TB cases reported in that study were caused by infections with $M$. tuberculosis (98.8\%) and $M$. africanum (1.2\%) respectively. In the tropical rainforest zone (Centre Region), 71 isolates were subjected to spoligotyping, 17 discrete spoligotypes of M. tuberculosis were detected. A total of $59(83 \%)$ isolates were grouped into five clusters (LAM10_CAM, Haarlem, T1, Uganda I, T2) whereas 12 (17\%) presented a single spoligotype each. The 17 Spoligotypes were compared with those contained in the international spoligotyping database (SpolDB4) and it was found that 61 isolates were already described in SpolDB4 while 10 were new or unique. In the savannah mosaic zone (West region and North-West region), spoligotyping showed that 25 patterns were detected among the 98 isolates. A total of $73(74.4 \%)$ isolates were grouped into 7 clusters (LAM10_CAM, Haarlem3, T1, T2, T5, LAM1, Haarlem1), whereas $25(25.6 \%)$ presented a single spoligotype. The 98 isolates were compared with those contained in the international spoligotyping database (SpolDB4). A total of 78 isolates were already described in SpolDB4 while 20 were new and unique It can be seen that the largest cluster of 33 (33.6\%) belonged to the Latin American-Mediterranean (LAM) family and exclusively to the type called LAM10_CAM. Other prominent strains included the Cameroon family (spoligotype ST 61), the Ghanian and Harlem spoligotypes. One single strain is designated in the SpolDB4 database as spoligotype 450 called Uganda I family and another as spoligotype 332 called West African 1 family. The clustering rate of the spoligotyping was 0.52 . MIRU-VNTR analysis was performed in both ecological zones on all the $169 \mathrm{M}$. tuberculosis isolates by using 24 published markers, which included 12 MIRU, 3 ETR, 7 QUB and 2 VNTR loci. The 169 isolates were all distributed into unique patterns (100\%). The clustering rate of the MIRU-VNTR was 0.014 for the tropical rainforest zone and 0 for the savannah mosaic zone. The allelic diversity $(\mathrm{h})$ differed for the individual loci, ranging from 0.01 to 0.75 for all zones investigated. The MIRU40, QUB 26 and QUB11b loci showed the highest discriminatory power $(\mathrm{h}=0.74)$, ETRB, QUB26, QUB4156 also showed the high allelic diversity $(\mathrm{h}=$ 0.6-0.68), and five other loci (MIRU 2, MIRU4, MIRU 23, MIRU31, and MIRU39, Mtub 34) showed low discriminating power $(\mathrm{h}<0.2)$. The other supplemental locus had a relatively high allelic diversity $(0.2<\mathrm{h}<0.6)$. The combination of the spoligotyping and MIRU-VNTR data revealed a further resolution of some of the clustered isolates identified by spoligotyping alone [29]. 
Another work done by Kamgue et al. on specimens collected from Centre region of Cameroon showed that Spoligotyping was performed by PCR-amplification followed by the reverse hybridization of 298 cultured specimens. Spoligotypes patterns were identified by comparison to reference strains in SPolDB4 database via the MIRU VNTR plus web application. About $97.65 \%$ of all tuberculosis (TB) cases were attributed to M. tuberculosis. A total of 65 different profiles were identified. Of these, 40 were represented as Shared Types (ST) while the others were orphans. LAM10_CAM and Haarlem families were the most prevalent genetic families with $51.01 \%$ and $14.09 \%$ respectively. ST 61, a member of the LAM10_ CAM family formed the largest cluster with 128 (42.95\%) isolates. No association was found between genotypes with regard to drug resistance and HIV sero-status. However, there was a significant association between genotypes and age groups. Patients belonging to 15-24 and 35-44 age groups were more likely infected by LAM10_CAM strains compared to others. The population structure of Mycobacterium tuberculosis complex strains from the Centre region was found to be diverse and the spoligotype 61 of the LAM10_CAM family was highly pre-dominant. Isolates of the LAM10_CAM seem to be not associated with drug resistance [7]. Koro Koro et al. showed in another previous work that M. africanum appears to be really polymorphic. The Afri- 2 family previously reported as a majority in West African countries counted 15 strains [30]. The 546 strains identified as $M$. tuberculosis were split into 81 different spoligotypes. Of the 565 MTBC strains, a total of 260 strains belonged to the Cameroon family, representing $45.9 \%$ and $47.6 \%$, respectively, of the $M$. tuberculosis isolates, compared with $43 \%$ and $47 \%$, respectively, found in the late 1990s [28]. The largest cluster of this family was formed by 186 isolates grouped in ST 61. The other commonly described lineages were Haarlem (103 isolates), T1 (55 isolates), and T2 (48 isolates), which together showed an increasing trend, shows the evolution of the MTBC population over a period of 7 years. None of the strains presented the spoligotype of the worldwide-expanded epidemic Beijing family; one strain was identified as M. bovis. The Cameroon family, as suggested by different studies on the Beijing family [31-33], could also have some selective advantages over other M. tuberculosis genotypes present in Cameroon, including virulence, pathogenesis, and epidemiologic characteristics. The table below give a summary of the genotyping of Mycobacterium tuberculosis complex from some important studies showing a diverse distribution of Mycobacterium tuberculosis species with the predominant spoligotypes as Cameroon family, Haarlem family and T1 clade in the investigated regions compared with previous work where it as not easy to determine new sub species (spoligotypes) because only the biochemical tests were used to study the diversity (Table 2).

\section{Drug Sensitivity of Tb Isolates and the Emergence of MDR in Cameroon}

Between 1997/98 and 2004/05 two drug hospital-based resistance surveys were conducted in the West Region of Cameroon. Sampling followed the protocol, recruiting systematically all consecutive new and retreatment cases during one year. The result shows the evolution of the anti-TB drug resistance profiles during a 7-years period, separated for new patients and for re-treatment patients. Looking at the trends of resistance patterns we observe a) a statistically insignificant decrease of initial and acquired overall resistance and b) a statistically insignificant rise of initial and acquired MDR. The decrease is principally due to the decrease of resistance to $\mathrm{S}$ (initial resistance) and $\mathrm{H}$ and $\mathrm{S}$ (acquired resistance) [31].

We further observed a very pronounced decrease of the proportion of re-treatment cases (23\% in $1997 / 98$ versus $8 \%$ in $2004 / 05)$ which means a decrease of the total reservoir of resistance. If we extrapolate these figures to the rest of the country we can expect annually between $2-4 \%$ of new $\mathrm{sm}+$ cases or at least about 280 patients to be primary resistant MDR-TB cases [31].

For the emergence of drug resistant $\mathrm{TB}$ in Cameroon several reasons may be evoked: Firstly, the economic crisis of the country during the late 1980's and the beginning of the 1990'ss resulted in a complete decline of the TB program which hitherto provided aa well organized and free diagnosis treatment and follow up of TB cases before the crisis. Diagnosis, treatment and follow up of TB patients became the individual responsibility of the treating physicians. As such the treatment of TB patients was disorganized until the reactivation of the National TB Program (NTP) from 1996 onwards, which attained covered the entire national territory only six years later in in 2002. Secondly, primary and secondary resistance to the first line drugs rifampicin and isoniazid (INH) to MTB isolates from Cameroonian TB patients arose and reached about $12 \%$ and $40 \%$, respectively, based on data from 2004-05 from the West Region. Additional resistance to rifampicin (R) can develop during treatment with the actual treatment scheme. Thus we can consider the rise of MDR-TB to have resulted from the shift from an older type of treatment regimen where $\mathrm{R}$ was not used continuously throughout the treatment period. Thirdly, treatment regimens were not standardized and physicians were left on their own to implement whatever treatment regimen they considered effective. Several specialists, not being associated with the NTP, experimented with repeated re-treatment schemes, sometimes associating thioacetazone - however without curing their patients. Fourthly, as treatment for MDR-TB was not readily available due to geographic, financial and health system constraints. MDR-TB patients

\begin{tabular}{|c|c|c|c|}
\hline $\begin{array}{l}\text { Regions of } \\
\text { Cameroon }\end{array}$ & Genotyping methods used & Findings & Reference \\
\hline West & spoligotyping & $\begin{array}{l}\text { M. tuberculosis: } 91 \%, \text { M. africanum: } 66 \% \\
\text { Cameroon family: } 34 \% \text {, Haarlem family: } 11 \% \text { ubiquitous spoligotype } 53 \text { (Ghanaian family): } \\
4.5 \%\end{array}$ & {$[27]$} \\
\hline West & $\begin{array}{l}\text { Spoligotyping, MIRU-VNTR, } \\
\text { IS6110- RFLP }\end{array}$ & $\begin{array}{l}\text { Confirmation of recent expansion of Cameroon family and the interpretation of molecular } \\
\text { typing results has to be adapted to the characteristics of the strain population within each } \\
\text { setting. }\end{array}$ & [28] \\
\hline Centre and West & Spoligotyping, MIRU-VNTR & $\begin{array}{l}\text { Mycobacterium tuberculosis: } 98.8 \%, \text { M. africanum: } 1.2 \% \\
\text { Haarlem family:7.6\%, Cameroon family: } 34.4 \% \text { and T clade: } 26.7 \%\end{array}$ & [30] \\
\hline West & Spoligotyping, MIRU-VNTR & $\begin{array}{l}\text { LAM10_CAM: } 51.01 \% \text {, Haarlem family: } 14.09 \% \text { ST } 61 \text { (Cameroon family): } 128 \text { (42.95\%) } \\
\text { isolates }\end{array}$ & [31] \\
\hline West & Spoligotyping & $\begin{array}{l}\text { M. tuberculosis: } 96.7 \%, \text { M. africanum: } 3.3 \% \\
\text { Cameroon family: } 34 \% \text {, Haarlem family: } 18.86 \% \text { ubiquitous T1: } 10 \% \text {; ubiquitous T2: } 8.79 \%\end{array}$ & [32] \\
\hline
\end{tabular}

Table 2: Genotyping results of Mycobacterium tuberculosis complex in Cameroon. MIRU-VNTR: Mycobacterial Interspersed Repetitive-Unit-Variable-Number TandemRepeat; IS6110-RFLP: Insertion Sequence 6110-Restriction Fragment Length Polymorphism. 
without treatment transmitted their germs to their environment thereby excercebating the crises. Fifthly, noncompliance with treatment regimens favoured the emergence and spread of MDR-TB [31].

The emergence of anti-tuberculosis drug resistance is a serious problem for TB control program in industrialized and developing countries alike. A global project on anti-tuberculosis drug resistance surveillance by the $\mathrm{WHO}$ and the International Union Against Tuberculosis and Lung Disease (IUATLD) [1] reported a prevalence of initial drug resistance of more than $10 \%$ in over 30 countries; it also identified 14 countries in which the prevalence of initial multidrug resistant TB strains (MDR-TB) (defined as resistance to at least isoniazid [INH] and rifampicin [RMP]) was more than 3\%. In a 2000 WHO report on Africa, the overall level of initial resistance varied from $6.3 \%$ to $24.8 \%$, and the level of MDR-TB from $1 \%$ to $5.3 \%$ [32]. However, the data available on the rates of drug resistance in Africa are not extensive and up to date. In Cameroon, a country with over 18 million inhabitants distributed in 10 regions (previously provinces), the incidence of tuberculosis is relatively high, with more than 300 new cases per 100,000 inhabitants yearly [33]. Further contribution to the increased death rate due to TB in the country has been the emergence of drug resistant strains to nearly all first line drugs [34]. RIF, INH, SM, and EMB are components of first-line multidrug therapy in Cameroon [4]. Between 1997 and 1998 in the West region of Cameroon, the overall resistance rate to at least one anti-TB drug was $26.9 \%$, with initial resistance being $19.7 \%$ and acquired resistance $51.1 \%$ [34]. In the Centre region of the country, the rate of initial resistance to at least one drug in 1995 was $31.8 \%$, and in 1998 it was $35.2 \%$ [26,35].

To determine the prevalence of acquired resistance (ADR) to the main anti-tuberculosis drugs and to identify risk factors associated with its occurrence in Yaounde. A total of 111 previously treated adults admitted consecutively to the tuberculosis centre with sputum smear-positive pulmonary tuberculosis between June 1996 and July 1997 were included in the study done by Kuaban et al. [36]. Growth of M. tuberculosis complex was obtained from sputum specimens of $98(88.3 \%)$ of the 111 patients studied; $57(58.2 \%)$ of these were resistant to at least one anti-tuberculosis drug. Resistance to isoniazid was the most common (54.1\%), followed by resistance to rifampicin (27.6\%), streptomycin (25.5\%) and ethambutol (12.2\%). Multidrug resistance was observed in $27(27.6 \%)$ of the cases. In a multivariate logistic regression analysis, ADR was significantly associated only with monotherapy used in previous tuberculosis treatment(s). The rate of acquired resistance was determined in the Centre region to be 58.2\% [6]. Another study in 15 district hospitals of the West region of Cameroon by Kuaban et al. between July 1997 and June 1998. To determine the prevalence of initial and acquired resistance to the main anti-tuberculosis drugs 2 years after the implantation of a tuberculosis control program in the region. Testing of susceptibility to the major anti-tuberculosis drugs was performed by the indirect proportion method. Growth of Mycobacterium tuberculosis complex strains was obtained from specimens of 566 (92\%) of the 615 patients. The overall resistance rate (one or more drugs) was $26.9 \%$, with initial resistance being $19.7 \%$ (86/437) and acquired resistance 51.1\% (66/129). Initial resistance to isoniazid was the most common (12.1\%), followed by streptomycin (11.7\%), ethambutol (2.5\%) and rifampicin $(2.1 \%)$. Initial resistance was noted as $13.5 \%$ to one drug, $4.3 \%$ to two, $1.1 \%$ to three and $0.7 \%$ to four. Acquired resistance to isoniazid was the most frequent (41.1\%), followed by streptomycin (26.4\%), rifampicin (14.7\%) and ethambutol (9.3\%). Acquired resistance was $25.6 \%$ to one drug, $14.7 \%$ to two, $7 \%$ to three and $3.9 \%$ to four. The proportion of resistant tuberculosis in the West Province was quite high. This underscores the need for the improvement of the control program by introducing the DOTS strategy [37].

Data on the levels of resistance of Mycobacterium tuberculosis complex (MTBC) strains to first line anti-tuberculosis drugs in Cameroon, and on the species of MTBC circulating in the country became obsolete 10 years after the last studies, and 6 years after the re-organisation of the National Tuberculosis (TB). A survey by Assam et al. from February to July 2009 in the West and Centre regions of Cameroon showed that of the 756 suspected patients, $154(20.37 \%)$ were positive by smear microscopy and among these, $16(10.70 \%)$ were resistant to at least one drug (13.30\%) for the West region and $8.10 \%$ for the Centre). The initial resistance rates were $7.4 \%$ for the Centre region and $11.30 \%$ for the West region, while the acquired resistance rates were $16.66 \%$ (1/6) for the Centre region and $23.07 \%$ (3/13) for the West. Within the two regions, the highest total resistance to one drug was obtained with INH and SM (2.68\% each). Multidrug-resistance (MDR) was observed only in the West region at a rate of $6.67 \%$. No resistance was recorded for EMB [13] on the same idea a work done in South, East regions showed that $11.9 \%$ were resistant to at least one drug. The initial resistance rate was $13.7 \%$ for the both regions, while no acquired resistance was found. Multidrug-resistance (MDR) was observed only in the South region where it was detected in $1.58 \%(1 / 63)$ isolates. No resistance was recorded for ethambutol (EMB) in the two regions $[38,39]$.

A retrospective cohort study was conducted in the Littoral Region of Cameroon in 2009 by Jurgen Noeske et al. including all sputum smear positive $(\mathrm{SM}+)$ PTB cases registered for retreatment. TB cases were identified and classified according to World Health Organization (WHO) recommendations for national $\mathrm{TB}$ programs. Bacterial susceptibility testing to first-line anti-TB drugs was performed using standard culture methods. In 2009, 5,668 TB cases were reported in the Littoral Region, of which 438 (7.7\%) were SM + PTB retreatment cases. DST results were available for $216(49.4 \%)$ patients. Twenty six patients (12\%) harbored multi-drug resistant (MDR) strains. Positive treatment outcome rates were particularly low in retreatment patients with MDR-TB (46.2\%; 95\% CI: 27.1-66.3). Thirteen MDRTB patients were successfully treated using a standardized MDR treatment regimen [40].

Kamgue et al. conducted a cross-sectional study from April 2010 to March 2011 in the Jamot Hospital in Yaounde showed that, from a total of 665 smear positive pulmonary tuberculosis patients enrolled. The overall resistance rate was $10.9 \%(63 / 576)$. The overall resistance rates for single drug resistance were: isoniazid-resistance 4.7\% (27/576), streptomycin-resistance $3.3 \%$ (19/576), rifampicin-resistance $0.2 \%$ (1/576), kanamycin-resistance $0.2 \%(1 / 576)$ and ofloxacin-resistance $0.2 \%(1 / 576)$. The MDR rate was $1.1 \%(6 / 576)$ and no extensively drug resistant tuberculosis (XDR) was detected [41].

\section{TB drug resistance mechanisms}

Major advances in molecular biology and the availability of new information generated after deciphering the complete genome sequence of $M$. tuberculosis [42], have led to the development of new tools for rapid detection of drug resistance $[43,44]$. Molecular methods are based on assigning the presence or absence of certain mutations in specific positions or genetic locations which are known to be associated with resistance [45]. About $95 \%$ of rifampicin (RIF)-resistant strains have mutations in the 81-bp core region of the rpoB gene encoding the $\beta$-subunit of the RNA polymerase, named RIF-Resistance Determining Region (RRDR) [46]. In contrast to RIF, the situation for isoniazid 
(INH) is much more complex. Resistance mutations have been reported in at least 4 different genes including katG, inhA, ahpC and oxyR [13]. Meanwhile, resistance against streptomycin (SM) has been reported to be associated with mutations in rrs gene, which codes for $16 \mathrm{~S}$ ribosomal RNA, and rpsL coding for the ribosomal protein S12 [47] and these mutations are found in a limited proportion of clinically isolated SM-resistant $M$. tuberculosis strains. Recently, Okamoto et al. [48] found that mutations within the gidB gene which encodes a conserved 7-methylguanosine $(\mathrm{m} 7 \mathrm{G})$ methyltransferase specific for the $16 \mathrm{~S}$ rRNA, is associated with low-level SM-resistance and are an important cause of resistance found in 33\% of resistant $M$. tuberculosis isolates. Resistance to ethambutol (EMB) is primarily mediated by mutations in the embB gene, coding for an arabinosyl transferase participating in mycobacterial cell wall synthesis, with codon 306 being most frequently affected [49]. Furthermore, mutations in the embA [50,51] and upstream of embC [52-54] are also involved in EMBresistance. A work done by Tekwu et al. a total of 63 drug resistant and 100 drug sensitive Mycobacterium tuberculosis complex clinical isolates were screened for genetic mutations in katG, inhA, ahpC, rpoB, rpsL, rrs, gidB and embCAB loci using DNA sequencing. Of the 44 isoniazid resistant (INHR) isolates ( 24 high level, $1 \mu \mathrm{g} / \mathrm{ml}$ and 20 low level, $0.2 \mu \mathrm{g} / \mathrm{ml}), 73 \%(32 / 44)$ carried the katG315 and/or the -15 inhA promoter mutations. Of the 24 high level INHR, 17 (70.8\%) harbored katG315 mutation, 1 a point mutation $(-15 \mathrm{C} \rightarrow \mathrm{T})$ in the inhA promoter and 6 were $(25.0 \%)$ wild types. Thus, for INHR high level detection, katG315 mutation had specificity and a sensitivity of $100 \%$ and $70.8 \%$ respectively. Of the 20 low level INHR, $10(50.0 \%)$ had a $-15 \mathrm{C} \rightarrow \mathrm{T}$ mutation in the inhA promoter region, and $1(2.2 \%)$ a $-32 \mathrm{G} \rightarrow \mathrm{A}$ mutation in the ahpC promoter region. All of the 7 rifampicin resistant (RIFR) isolates carried mutations in the rpoB gene (at codons Ser531Leu (71.4\%), His526Asp (14.3\%), and Asp516Val (14.3\%)). Of the 27 streptomycin resistant (SMR) isolates, 7 carried mutations at the rpsL and the gidB genes. 1 of the 2 ethambutol resistant (EMBR) isolates displayed a mutation in embB gene. Table 3 presents a summary of resistance profile of Mycobacterium tuberculosis in different selected regions of Cameroon showing a predominance resistance to isoniazid and streptomycin with a small decrease of MDR and no XDR registered (Table 3).

\section{Control of $\mathrm{Tb}$ and the National Anti-Tb Control Program}

The history of tuberculosis control in Cameroon from 2009 back to 1980 can be divided into three main periods. The first period, from 1980 to 1994, corresponded to the implementation of the 'primary health care' policy. At that time, tuberculosis case management was delivered free of charge, but centralized in specialized services with a gradual and mild increase in new cases detected. The second period, from 1995 to 2000 , was characterized by the implementation of the 'primary health care reorientation' policy that decentralized tuberculosis care to all health facilities, but introduced cost recovery --which came along with a dramatic drop in the number of tuberculosis cases detected. The National tuberculosis control program, established in 1996, entrusted health facilities--especially hospitals--with the responsibility of tuberculosis diagnosis and treatment, and referred to them as tuberculosis diagnosis and treatment centers. During the third period, from 2001 to 2009 , owing to major support from global health initiatives, the number of tuberculosis diagnosis and treatment centers was increased (reaching 216 centers in 2009), with a significant increase of new cases detected that peaked in 2006, from where the situation started declining till 2009 [35].

\section{Current management protocol for MDR-TB cases in cameroon}

Currently MDR-TB cases are referred to and manged within the TB control programme Lung specialists, public health experts, representatives of the NTP, of the Ministry of Healthe $(\mathrm{MoH})$ and of the National Reference Laboratory adopted a treatment scheme together with technical guidelines. Since 2005, 19 three specialized units are in charge of MDR-TB diagnosis and treatment: Jamot TB Reference Hospital in Yaoundé (Jamot), the Centre of PneumoPhtysiology of Laquintinie Hospital (Laquintinie) and Dibamba Catholic Health Centre (Dibamba). Failures among retreatment cases are referred to Jamot or Laquintinie or Dibamba hospitals or are proposed to undergo sputum culture (in the National TB Reference Laboratory (CPC) in Yaounde or in CEBEC Baptist Hospital (CEBEC) in Douala. Drug Sensitivity tests (DST) for both laboratories are done in CPC. In case of culture-confirmed MDR-TB diagnosis, patients are taken care and a 'kit' at the office of health programme of the German Technical Cooperation (GTZ) in Douala is then delivered directly to and managed in one of the three specialized MDR-TB treatment units. GTZ is in charge of the drug procurement. The GTZ office imports the drugs. Prices vary currently between 300 USD and 500 USD according to patients' weight [35].

\section{Strategies and methods for case finding and contact tracing}

TB control relies on passive case detection through the general and primary health care (PHC) services. Sputum examinations are performed for all suspected TB cases in the DTCs either for suspects

\begin{tabular}{|c|c|c|}
\hline $\begin{array}{c}\text { Regions } \\
\text { investigated }\end{array}$ & Findings & Reference \\
\hline Centre & $\begin{array}{l}\text { Resistance to isoniazid: } 54.1 \% \text {, to rifampicin: } 27.6 \% \text {, streptomycin } 25.5 \% \text {; ethambutol: } 12.2 \% \text {; Multidrug resistance: } 27.6 \% \text {, acquired } \\
\text { resistance: } 58.2 \% \text {. }\end{array}$ & [40] \\
\hline West & $\begin{array}{l}\text { overall resistance rate: } 26.9 \% \text {, initial resistance: } 19.7 \% \text { and acquired resistance: } 51.1 \% \\
\text { Initial resistance to isoniazid: } 12.1 \% \text {, streptomycin: } 11.7 \% \text {, ethambutol: } 2.5 \% \text { and rifampicin: } 2.1 \% \text {. } \\
\text { Acquired resistance to isoniazid: } 41.1 \% \text {, streptomycin: } 26.4 \% \text {, rifampicin: } 14.7 \% \text { and ethambutol: } 9.3 \% \text {. }\end{array}$ & [41]. \\
\hline Centre & resistant to at least one drug: $8.1 \%$; initial resistance rates: $7.35 \%$ acquired resistance rates: $16.66 \%$; No MDR & [42] \\
\hline West & $\begin{array}{l}\text { resistant to at least one drug: } 13.3 \% \text {; initial resistance rates: } 11.29 \% \text {; acquired resistance rates: } 23.07 \% \text {; Multidrug-resistance } \\
\text { (MDR): } 6.67 \% \text {. }\end{array}$ & [42] \\
\hline South end East & $\begin{array}{l}\text { resistant to at least one drug: } 11.9 \% \text {; initial resistance rate: } 13.7 \% \text {; no acquired resistance; Multidrug-resistance (MDR): } 1.58 \% \text {; No } \\
\text { resistance for ethambutol }\end{array}$ & {$[43]$} \\
\hline Littoral & Twenty six patients (12\%) harbored multi-drug resistant (MDR) strains. & [44] \\
\hline Centre & $\begin{array}{l}\text { The overall resistance: } 10.9 \% \text {; The overall resistance for isoniazid: } 4.7 \% \text {, streptomycin: } 3.3 \% \text {, rifampicin: } 0.2 \% \text {, kanamycin: } 0.2 \% \\
\text { and ofloxacin: } 0.2 \% \text {. The MDR rate: } 1.1 \% \text { and no XDR }\end{array}$ & [45]. \\
\hline
\end{tabular}

Table 3: Summary of resistance profiles of Mycobacterium tuberculosis in different regions of Cameroon. MDR: Multidrug-Resistance; XDR: Extensively Drug Resistant Tuberculosis. 
presenting directly in one of the DTCs or for referred suspects. Algorithms for the detection of ss-PTB cases are conceived and applied according to WHO's and the European Union's recommendations. Regularly, awareness campaigns are performed through the media in order to diffuse knowledge about TB signs and symptoms, the localization of DTCs and the conditions for being diagnosed and treated. Some Regions produce and distribute continuously flyers with information about $\mathrm{TB}$ and $\mathrm{TB}$ treatment facilities, too, for special populations like prison inmates. In some Christian missionary networks, routine contact tracing among family members is done during systematic home visits of TB patients. - Active case finding for under-five children of infectious cases is recommended in the national guidelines but still not applied systematically [35].

\section{Conclusions}

Investigations of genetic polymorphism of M. tuberculosis complex strains from humans in Cameroon has shown M. tuberculosis sensu stricto to be the predominant agent of TB cases, with the preponderance of the LAM10 family genotype and large shift of M. africanum. The resistance to all first line anti-TB drugs has declined significantly, however, the general rates of anti-TB drug resistance remain high in some regions, underscoring the need for greater enforcement of control strategies in the country.

\section{Acknowledgements}

The experimental work of the authors cited herein received financial support from the Biosciences Eastern and Central African Network (BeCA) and the International Programme in the Chemical Sciences (IPICS), Uppsala University.

\section{References}

1. World Health Organization (2013) Global tuberculosis report 2013

2. Nola D (2014) TB in Cameroon-Control efforts and the way forward

3. Huet M, Rist N, Boube G, Potier D (1971) Bacteriological study of tuberculosis in Cameroon. Rev Tuberc Pneumol 35: 413-426.

4. Niobe-Eyangoh SN, Kuaban C, Sorlin P, Cunin P, Thonnon J, et al. (2003) Genetic biodiversity of Mycobacterium tuberculosis complex strains from patients with pulmonary tuberculosis in Cameroon. J Clin Microbiol 41: 2547 2553.

5. Warren R, de Kock M, Engelke E, Myburgh R, Gey van Pittius N, et al. (2006) Safe Mycobacterium tuberculosis DNA extraction method that does not compromise integrity. J Clin Microbiol 44: 254-256.

6. Assam- Assam AJP, PenlapVB, Cho-Ngwa F, Tedom JC, Anyangwe I, et al. (2011) Mycobacterium tuberculosis complex drug resistance pattern and identification of species causing TB in the West and Centre regions of Cameroon. BMC Infectious Diseases 11: 94

7. Sidze LK, Tekwu EM, Kuaban C, Assam-Assam JP, Tedom JC, et al. (2013) Estimates of Genetic Variability of Mycobacterium tuberculosis Complex and Its Association with Drug Resistance in Cameroon. Advances in Infectious Diseases 3: 55-59.

8. Awah-Ndukum J, Kudi AC, Bah GS, Bradley G, Tebug SF, et al. (2012) Bovine tuberculosis in cattle in the highlands of Cameroon: seroprevalence estimates and rates of tuberculin skin test reactors at modified Cut-OffsVet. Med Int 2012 13.

9. Awah-Ndukum J, Tchoumboue J, Niba AT (2005) Prevalence of bovine tuberculosis at the SODEPA Douala abattoir, Cameroon (1995-2003). Cameroon Journal of Experimental Biology 2012: 116-120.

10. Awah-Ndukum J, Kudi AC, Bradley G, Ane-Anyangwe IN, Fon-Tebug S, et al. (2010) Prevalence of bovine tuberculosis in abattoirs of the Littoral and Western highland regions of Cameroon: A cause for public health concern. Veterinary Medicine International.

11. Awah-Ndukum J, Kudi AC, Bradley G, Ane-Anyangwe I, Titanji VPK, et al (2012) Prevalence of bovine tuberculosis in cattle in the highlands of Cameroon based on the detection of lesions in slaughtered cattle and tuberculin skin tests of live cattle. Veterinary Medicine International 57: 59-76.
12. Koro FK, Bouba, Foko E, Ngatchou AF, Eyangoh S, et al. (2013) First insight into the current prevalence of bovine tuberculosis in cattle slaughtered in cameroon: the case of main abattoirs of yaounde and douala. British Microbiology Research Journal 3: 272-279.

13. (2006) World Health Organization: Global tuberculosis control: surveillance, planning and financing. Publication WHO/HTM/2006.362 Geneva, Switzerland

14. (2014) World Health Organization: Global tuberculosis control: WHO Report 2014. Publication WHO/HTM/TB/2014.17 Geneva, Switzerland.

15. Kamerbeek J, Schouls L, Kolk A, van Agterveld M, van Soolingen D, et al. (1997) Simultaneous detection and strain differentiation of Mycobacterium tuberculosis for diagnosis and epidemiology. J Clin Microbiol 35: 907-914.

16. Mazars E, Lesjean S, Banuls AL, Gilbert M, Vincent V, et al. (2001) Highresolution minisatellite-based typing as a portable approach to global analysis of Mycobacterium tuberculosis molecular epidemiology. Proc Natl Acad Sci USA 98: 1901-1906.

17. Le Flèche P, Fabre M, Denoeud F, Koeck JL, Vergnaud G (2002) High resolution, on-line identification of strains from the Mycobacterium tuberculosis complex based on tandem repeat typing. BMC Microbiol 2: 37.

18. Sola C, Filliol I, Legrand E, Mokrousov I, Rastogi N (2001) Mycobacterium tuberculosis phylogeny reconstruction based on combined numerical analysis with IS108, IS6110, VNTR, and DR-based spoligotyping suggests the existence of two new phylogeographical clades. J Mol Evol 53: 680-689.

19. Filliol I, Driscoll JR, Van Soolingen D, Kreiswirth BN, Kremer K, et al. (2002) Global distribution of Mycobacterium tuberculosis spoligotypes. Emerg Infect Dis 8: 1347-1349.

20. Sola C, Filliol I, Gutierrez MC, Mokrousov I, Vincent V, et al. (2001) Spoligotype database of Mycobacterium tuberculosis: biogeographic distribution of shared types and epidemiologic and phylogenetic perspectives. Emerg Infect Dis 7 : 390-396.

21. Allix C, Supply P, Fauville-Dufaux M (2004) Utility of fast mycobacteria interspersed repetitive unit-variable number tandem repeat genotyping in clinical mycobacteriological analysis. Clin Infect Dis 39: 783-789.

22. Kwara A, Schiro R, Cowan LS, Hyslop NE, Wiser MF, et al. (2003) Evaluation of the epidemiologic utility of secondary typing methods for differentiation of Mycobacterium tuberculosis isolates. J Clin Microbiol 41: 2683-2685.

23. Evans JT, Hawkey PM, Smith EG, Boese KA, Warren RE, et al. (2004) Automated high-throughput mycobacterial interspersed repetitive unit typing of Mycobacterium tuberculosis strains by a combination of PCR and nondenaturing high-performance liquid chromatography. J Clin Microbiol 42 : 4175-4180.

24. Supply PC, Allix S, Lesjean M, Cardoso-Oelemann S, Rusch-Gerdes E, et al. (2006) Proposal for standardization of optimized mycobacterial interspersed repetitive unit-variable-number tandem repeat typing of Mycobacterium tuberculosis. J Clin Microbiol 44: 4498-4510.

25. Oelemann MC, Diel R, Vatin V, Haas W, Rüsch-Gerdes S, et al. (2007) Assessment of an optimized mycobacterial interspersed repetitive- unitvariable-number tandem-repeat typing system combined with spoligotyping for population-based molecular epidemiology studies of tuberculosis. $\mathrm{J}$ Clin Microbiol 45: 691-697.

26. Soini H, Musser JM (2001) Molecular diagnosis of mycobacteria. Clin Chem 47: 809-814.

27. Niobe-Eyangoh SN, Kuaban C, Sorlin P, Thonnon J, Vincent V, et al. (2004) Molecular characteristics of strains of the Cameroon family, the major group of Mycobacterium tuberculosis in a country with a high prevalence of tuberculosis. J Clin Microbiol 42: 5029-5035.

28. Beng VNP, Victor T, Warren R, Jordaan A, Tedom, et al. (2010) Evidence of drug resistance among the LAM Cameroon family in mycobacterium tuberculosis isolates from Yaounde, Cameroon. JCAS 9: 11-15.

29. Assam-Assam JP, Beng VP, Cho-Ngwa F, Toukam M, Ngwe AAl, et al. (2013) Mycobacterium tuberculosis is the causative agent of tuberculosis in the southern ecological zones of $f$ Cameroon, as shown by genetic analysis. BMC Infectious Diseases 13:431.

30. Koro FK, Simo YK, Piam FF, Noeske J, Gutierrez C, et al. (2013) Population dynamics of tuberculosis bacilli in Cameroon as assessed by spoligotyping. $J$ Clin Microbiol 51: 299-302.

31. Brosch R, Gordon SV, Marmiesse M, Brodin P, Buchrieser C, et al. (2002) A 
Citation: Titanji VPK, Assam JPA (2016) Drug Sensitivity and Molecular Diversity of M. tuberculosis in Cameroon: A Meta-analysis. J Vasc Med Surg 4: 266. doi:10.4172/2329-6925.1000266

Page 8 of 8

new evolutionary scenario for the Mycobacterium tuberculosis complex. Proc Natl Acad Sci USA 99: 3684-3689.

32. Parwati I, van Crevel R, van Soolingen D (2010) Possible underlying mechanisms for successful emergence of the Mycobacterium tuberculosis Beijing genotype strains. Lancet Infect Dis 10: 103-111.

33. (2014) National Tuberculosis Strategic Plan 2010-2014.

34. (2000) WHO (World Health Organization): Anti-tuberculosis drug resistance in the world. Report number 2: Prevalence and Trends. The WHO/IUATLD global project on anti-tuberculosis drug resistance surveillance. WHO/CDS/ TB/2000.278 Geneva, Switzerland.

35. Kuaban C, Bercion R, Noeske J, Cunin P, Nkamsse P, et al. (2000) Antituberculosis drug resistance in the west province of Cameroon. Int $\mathrm{J}$ Tuberc Lung Dis 4: 356-360.

36. Bercion R, Kuaban C (1997) Initial resistance to antituberculosis drugs in Yaounde, Cameroon in 1995. Int $\mathrm{J}$ Tuberc Lung Dis 1: 110-114.

37. Bercion R, Kuaban C (1998) Resistance of mycobacterial tuberculosis complex to the main antibacillary agent in Yaounde, Cameroon. Bull Soc Pathol Exot 91: 199-202.

38. Assam Assam JP, PenlapVB, Cho-Ngwa F, Tedom JC, Anyangwe I, et al. (2011) Mycobacterium tuberculosis complex drug resistance pattern and identification of species causing TB in the south and east regions of Cameroon. Journal of Microbiology and Antimicrobials 3: 191-195.

39. Noeske J, Voelz N, Fon E, Abena JL (2012) Foe early results of systematic drug susceptibility testing in pulmonary tuberculosis retreatment cases in Cameroon. BMC Research Notes 5: 160.

40. Sidze LK, Tekwu EM, Kuaban C, Assam-Assam JP, Tedom JC, et al. (2014) Drug resistance tuberculosis decrease and no XDR as an indicator of the TB control program performance in the Central Region of Cameroon.

41. Cole ST, Brosch R, Parkhill J, Garnier T, Churcher C, et al. (1998) Deciphering the biology of Mycobacterium tuberculosis from the complete genome sequence. Nature 393: 537-544.

42. Holroyd NS, Hornsby T, Jagels K, Krogh A, McLean J, et al. (1998) Deciphering the biology of Mycobacterium tuberculosis from the complete genome sequence. Nature 393: 537-544.

43. Shamputa IC, Rigouts And L, Portaels F (2004) Molecular genetic methods for diagnosis and antibiotic resistance detection of mycobacteria from clinical specimens. APMIS 112: 728-752.

44. Boehme CC, Nabeta P, Hillemann D, Nicol MP, Shenai S, et al. (2010) Rapid molecular detection of tuberculosis and rifampin resistance. N Engl J Med 363 : 1005-1015.

45. García de Viedma D (2003) Rapid detection of resistance in Mycobacterium tuberculosis: a review discussing molecular approaches. Clin Microbiol Infect 9: 349-359.

46. Zhang Y, Yew WW (2009) Mechanisms of drug resistance in Mycobacterium tuberculosis. Int J Tuberc Lung Dis 13: 1320-1330.

47. Sreevatsan S, Pan X, Stockbauer KE, Williams DL, Kreiswirth BN, et al. (1996) Characterization of rpsL and rrs mutations in streptomycin-resistant Mycobacterium tuberculosis isolates from diverse geographic localities. Antimicrob Agents Chemother 40: 1024-1026.

48. Okamoto S, Tamaru A, Nakajima C, Nishimura K, Tanaka Y, et al. (2007) Loss of a conserved 7-methylguanosine modification in 16S rRNA confers low-leve streptomycin resistance in bacteria. Mol Microbiol 63: 1096-1106.

49. Plinke C, Rusch-Gerdes S, Niemann S (2006) Significance of mutations in embB codon 306 for prediction of ethambutol resistance in clinical Mycobacterium tuberculosis isolates. Antimicrob Agents Chemother 50: 1900-1902.

50. Ramaswamy S, Musser JM (1998) Molecular genetic basis of antimicrobia agent resistance in Mycobacterium tuberculosis: 1998 update. Tuber Lung Dis 79: 3-29.

51. Plinke C, Cox HS, Zarkua N, Karimovich HA, Braker K, et al. (2010) embCAB sequence variation among ethambutol-resistant Mycobacterium tuberculosis isolates without embB306 mutation. J Antimicrob Chemother 65: 1359-1367.

52. Jadaun GP, Das R, Upadhyay P, Chauhan DS, Sharma VD, et al. (2009) Role of embCAB gene mutations in ethambutol resistance in Mycobacterium tuberculosis isolates from India. Int J Antimicrob Agents 33: 483-486.

53. Dalla Costa ER, Ribeiro MO, Silva MS, Arnold LS, Rostirolla DC, et al. (2009) Correlations of mutations in katG, oxyR-ahpC and inhA genes and in vitro susceptibility in Mycobacterium tuberculosis clinical strains segregated by spoligotype families from tuberculosis prevalent countries in South America. BMC Microbiol 9: 39.

54. Tekwu EM, Sidze LK, Assam-Assam JP, Tedom JC, Tchatchouang S, et al. (2014) Sequence analysis for detection of drug resistance in Mycobacterium tuberculosis complex isolates from the Central Region of Cameroon. 